\title{
Exhaustion of the gas next to the supermassive black hole of M31
}

\author{
Anne-Laure Melchior ${ }^{1}$ and Françoise Combes $^{2}$ \\ ${ }^{1}$ LERMA, Observatoire de Paris, PSL Research University, CNRS, Sorbonne Universités, UPMC Univ. Paris 06, 75014, Paris, \\ France \\ e-mail: A.L.Melchior@obspm.fr \\ 2 Collège de France, 11 Place Marcelin Berthelot, 75005 Paris, France \\ e-mail: Francoise.Combes@obspm.fr
}

Received 30 March 2017 / Accepted 24 September 2017

\begin{abstract}
New observations performed at the IRAM Plateau de Bure reveal the absence of molecular gas next to the black hole of the Andromeda galaxy. We derived a $3 \sigma$ upper limit on the molecular gas mass of $4300 M_{\odot}$ for a line width of $1000 \mathrm{~km} \mathrm{~s}^{-1}$. This is compatible with infra-red observations, which reveal a hole in the dust emission next to the black hole. Some gas from stellar feedback is expected from the old eccentric stellar disc population, but it is not accreted close to the black hole. This absence of gas explains the absence of stellar formation observed in this region, contrary to what is observed next to Sgr A* in the Milky Way. Either the gas has been swallowed by the black hole, or a feedback mechanism has pushed the gas outside the central $1 \mathrm{pc}$. Nevertheless, we detect a small clump of gas with a very low velocity dispersion at $2.4^{\prime \prime}$ from the black hole. It is probable that this clumpy gas is seen in projection, as it does not follow the rotation of the disc surrounding the black hole, its velocity dispersion is ten times lower than the expected velocity gradient, and the tidal shear from the black hole requires a gas density for this clump that is not compatible with our observations.
\end{abstract}

Key words. galaxies: individual: M31 - galaxies: kinematics and dynamics - ISM: molecules - submillimeter: ISM

\section{Introduction}

Andromeda is a galaxy that lies in the green valley (Mutch et al. 2011; Tempel et al. 2011; Jin et al. 2014). According to Belfiore et al. (2016), it is typically a low-ionisation emissionline region (LIER), as first observed by Rubin \& Ford (1971) and discussed by Heckman (1996). González-Martín et al. (2015) discussed that the torus is disappearing in LIER: there is indeed little gas in the inner part of M31 (Melchior et al. 2000; Melchior \& Combes 2011, 2013). It is the closest external large galaxy in which we can explore the mechanisms that quenched the star formation activity. Optical ionised gas has been observed by Menezes et al. (2013) next to the black hole in a field of view ${ }^{1}$ of $5^{\prime \prime} \times 3.5^{\prime \prime}$, but this emission is weak. Jacoby et al. (1985) estimated the ionised gas mass in the bulge $\left(10^{\prime} \times 10^{\prime}\right)$ to be of the order of $1500 M_{\odot}$. It also hosts a very massive black hole of $1.4 \times$ $10^{8} M_{\odot}$ (Bender et al. 2005), but as studied by Li et al. (2011a), it is non-active and only murmurs at a level of $10^{-10} L_{\mathrm{Edd}}$. It hosts very little star formation of the order of $0.25-0.3 M_{\odot} \mathrm{yr}^{-1}$, mainly located in the $10 \mathrm{kpc}$ ring of the disc (e.g. Ford et al. 2013; Rahmani et al. 2016). Inside the central region $\left(10^{\prime} \times 10^{\prime}\right)$, no obvious sign of star formation is detected (e.g. Kang et al. 2012; Azimlu et al. 2011; Amiri \& Darling 2016), except for a central cluster of A stars formed $200 \mathrm{Myr}$ ago that is located next to the black hole (within $1^{\prime \prime}$ ) (Lauer et al. 2012), designated by P3 by Bender et al. (2005). Viaene et al. (2014) estimated the star formation rate (SFR) on a pixel basis with panchromatic spectral energy distribution modelling. This infrared-based SFR estimated in the central pixel $\left(36^{\prime \prime} \times 36^{\prime \prime}\right)$ is $4 \times 10^{-5} M_{\odot} \mathrm{yr}^{-1}$, while an integration over the central region with a radius of $1 \mathrm{kpc}$ corresponds to $1.25 \times 10^{-3} M_{\odot} \mathrm{yr}^{-1}$. This negligible SFR is much lower than the value predicted by Rimoldi et al. (2016),

\footnotetext{
1 For compatibility reasons, we assume a distance to M31 of $780 \mathrm{kpc}$
} throughout, that is, 1 arcsec $\sim 3.8 \mathrm{pc}$, following Melchior et al. (2000). considering supernovae remnants expected within the sphere of influence of quiescent supermassive black holes. For M31, an SFR of $0.13 M_{\odot} \mathrm{yr}^{-1}$ is expected in the sphere of influence $\left(R_{\mathrm{SOI}}=14 \mathrm{pc}=3.7^{\prime \prime}\right)$ of its supermassive black hole. A past AGN activity is also expected, and the associated molecular torus, if it survives, should have a radius $R_{\mathrm{MT}}=25 \mathrm{pc}=6.7^{\prime \prime}$. In parallel, Chang et al. (2007) expected next to the black hole an accumulation of molecular gas (about $10^{4} M_{\odot}$ ) originating from stellar feed-back. Melchior \& Combes (2013) estimated a minimum molecular mass of $4.2 \times 10^{4} M_{\odot}$ within $30^{\prime \prime}$ from the centre, while about $10^{6} M_{\odot}$ of gas is expected from stellar feedback (e.g. Gallagher \& Hunter 1981).

In this Letter, we present new observations of molecular gas with the IRAM Plateau de Bure interferometer (PdBI). We discuss the implications of the non-detection of gas next to the black hole.

\section{Observations}

The $3 \mathrm{~mm}$ observations were carried out at IRAM PdBI with the five-antenna configuration between 3 September and 24 December 2012 in C-array and D-array. The receivers were tuned to a frequency of $115.386 \mathrm{GHz}$, to account for the systemic velocity of M31 $\left(-300 \mathrm{~km} \mathrm{~s}^{-1}\right)$. We used the WideX correlator, which provides a broad frequency range of $3.6 \mathrm{GHz}$ and $2 \mathrm{MHz}$ spectral resolution. We thus probe a velocity range of $9000 \mathrm{~km} \mathrm{~s}^{-1}$. The integration time is about six hours per field, but for the field at the offset $\left(-16.8^{\prime \prime},-21.5^{\prime \prime}\right)$, which has been integrated, 34\% lower: as shown in Fig. 1, the level of noise is higher in the south-western part of the data cube. After calibration within the GILDAS reduction package, the visibility set was processed with the MAPPING software. A one-iteration CLEAN deconvolution was applied to recover a primary-beam-corrected data cube close to the signal. The beam size of the final data was $3.37^{\prime \prime} \times 2.44^{\prime \prime}$ (corresponding to $12.8 \mathrm{pc} \times 9.3 \mathrm{pc}$ ) with a position angle $\mathrm{PA}=70 \mathrm{deg}$, while the data are sampled with a pixel 


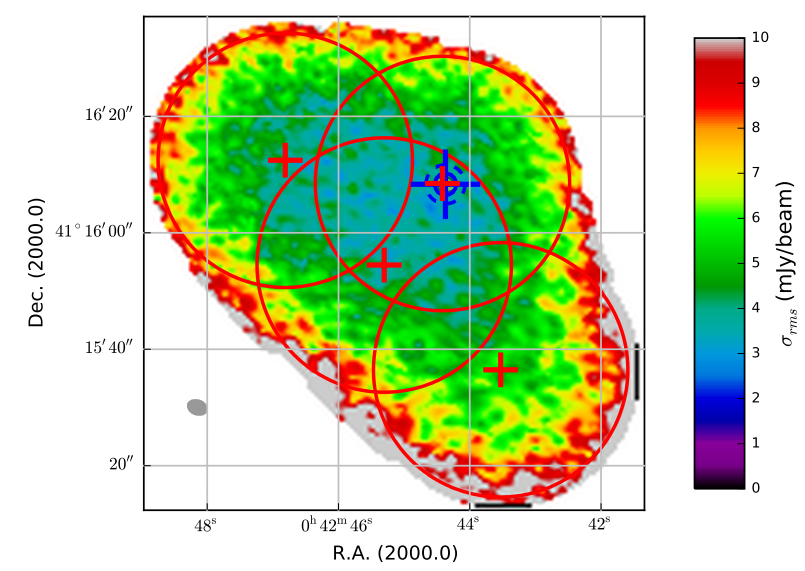

Fig. 1. IRAM-PdB $\mathrm{CO}(1-0)$ rms map. The colour-coding corresponds to the noise level $\left(\sigma_{\mathrm{rms}}\right)$, corrected for the primary beam, of the map obtained at the IRAM-PdB. The blue cross indicates the (optical) centre of Andromeda (Crane et al. 1992). The red crosses correspond to the centre of the four fields observed at PdB with a half-power primary beam of 43.7" represented by red circles. The half-power beam is presented in the bottom left corner of the $\mathrm{CO}(1-0)$ observations.

size of $0.61^{\prime \prime}$. At the black hole position, we reach a sensitivity of $3.2 \mathrm{mJy} /$ beam at $1 \sigma$ with $\Delta v=5.1 \mathrm{~km} \mathrm{~s}^{-1}$, and $0.6 \mathrm{mJy} /$ beam at $1 \sigma$ with $\Delta v=304 \mathrm{~km} \mathrm{~s}^{-1}$, as displayed in Fig. 2. The extremities of the band are more noisy, and hence, a more optimistic value is found using the radiometer formula with $0.4 \mathrm{mJy} / \mathrm{beam}$ at $1 \sigma$ with $\Delta v=304 \mathrm{~km} \mathrm{~s}^{-1}$. We subsequently restrict our analysis to the velocity range $(-300,+300) \mathrm{km} \mathrm{s}^{-1}$. Figure 1 displays the rms map obtained for each channel, where the signal has been removed.

\section{Analysis}

\subsection{No accumulation of gas next to the black hole}

If there were a gaseous disc/ring surrounding the black hole, as observed in the Milky Way (e.g. Dahmen et al. 1998; Kauffmann et al. 2017) and in external galaxies (Ricci et al. 2015), given the black hole mass and the stellar velocity dispersion, one would expect a molecular gas signal of $2 \mathrm{mJy}$ with a line width of about $1000 \mathrm{~km} \mathrm{~s}^{-1}$ according to Chang et al. (2007). This corresponds to a $10^{4} M_{\odot}$ accumulation of molecular gas next to the black hole due to stellar feedback from the eccentric inner stellar disc.

Following Solomon \& Vanden Bout (2005), we can derive the molecular hydrogen mass $M_{\mathrm{H}_{2}}=\alpha_{\mathrm{CO}} \times L_{\mathrm{CO}}^{\prime}$ with $\alpha_{\mathrm{CO}}=4.36 M_{\odot}\left(\mathrm{K} \mathrm{km} \mathrm{s}^{-1} \mathrm{pc}^{2}\right)^{-1}$, and the line luminosity, in $\mathrm{K} \mathrm{km} \mathrm{s}^{-1} \mathrm{pc}^{2}$ :

$L^{\prime}{ }_{\mathrm{CO}}=3.25 \times 10^{7} S_{\mathrm{CO}} \Delta V \times \frac{D_{L}^{2}}{v_{\text {rest }}^{2}} \frac{1}{(1+z)}$,

with $S_{\mathrm{CO}} \Delta V$ in Jy $\mathrm{km} \mathrm{s}^{-1}, D_{L}$ in Mpc and $v_{\text {rest }}$ in GHz. Relying on the level of noise we have reached, we estimate an $(3 \sigma)$ upper limit of the molecular gas mass in the beam of $4300 M_{\odot}$ $\left(2400 M_{\odot}\right)$ for a line width of $1000 \mathrm{~km} \mathrm{~s}^{-1}\left(300 \mathrm{~km} \mathrm{~s}^{-1}\right)$. As discussed above, these values can be increased by $40 \%(30 \%)$ with a direct smoothing of the large band data.

\subsection{Small gas clumps: residuals of a past molecular torus?}

We investigated the possibility that the gas might be present in the form of small clumps. Given the good velocity resolution

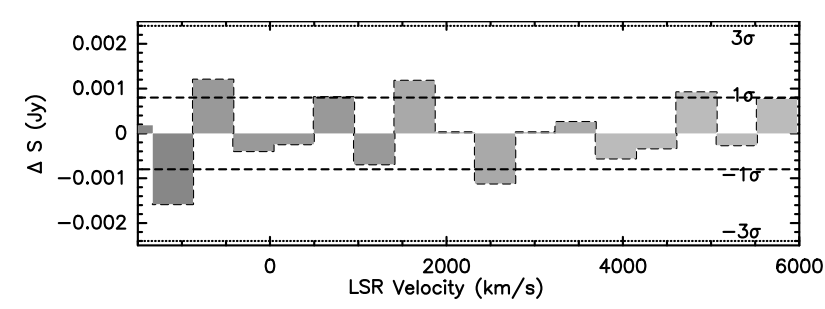

Fig. 2. Spectra of the central pixel smoothed at $304 \mathrm{~km} \mathrm{~s}^{-1}$. The dashed (dotted) lines correspond to the $1 \sigma(3 \sigma)$ interval, with $\sigma=0.6 \mathrm{mJy}$. The systemic velocity of M31 $\left(-300 \mathrm{~km} \mathrm{~s}^{-1}\right)$ has been removed.

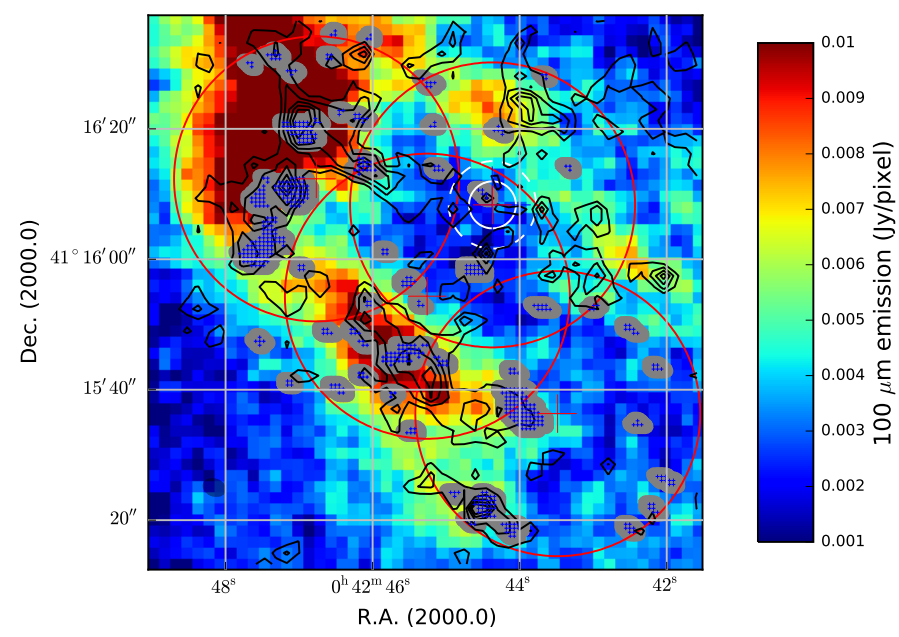

Fig. 3. Position of CO excess superimposed on $100 \mu \mathrm{m}$ Herschel/PACS map. The contours $(1.5,2.6,3.7,4.9$, and $6 \mathrm{MJy} / \mathrm{sr})$ correspond to the Spitzer $8 \mu \mathrm{m}$ map where the stellar continuum has been substracted by the adjustment of elliptical annuli using the standard surface photometry algorithm developed for IRAF (Jedrzejewski 1987). The projected field of view is $230 \mathrm{pc} \times 230 \mathrm{pc}$. Following Rimoldi et al. (2016), the white circle plotted with the dashed line corresponds to the expected extent of a molecular torus $\left(R_{\mathrm{MT}}=25 \mathrm{pc} \sim 6.7^{\prime \prime}\right)$, while the white circle plotted with the full line corresponds to the radius of the sphere of influence of the black hole $\left(R_{\mathrm{SOI}}=14 \mathrm{pc} \sim 3.7^{\prime \prime}\right)$, as displayed in Fig. 1 .

and the quality of the interferometric data, we looked for statistically significant weak signals, higher than $3 \sigma$ over a beam area, with the procedure described in Dassa-Terrier et al. (in prep.). The cores of the detected clumps are plotted in Fig. 3 as blue crosses, while the beam associated with each detected pixel is represented in grey.

We can note that there is an overall correlation with the dust emission detected at $100 \mu \mathrm{m}$ detected with Herschel/PACS, while $\mathrm{CO}$ exhibits a better correlation with the $8 \mu \mathrm{m}$ dust emission map from Spitzer, displayed as contours in Fig. 3. Next to the centre, the region of interest in this Letter, there is no dust emission at $100 \mu \mathrm{m}$. Within $12^{\prime \prime}$, there is only one clump with a peak flux above $6 \sigma$, which we discussed here. The other detections and their reliabilities will be discussed elsewhere (DassaTerrier et al., in prep.).

We extracted the spectrum of the remaining clump in GO VIEW in MAPPING/Gildas. We then analysed this spectrum with CLASS in Gildas. The results are provided in Table 1. We estimate a velocity dispersion $\sigma_{V}<5.9 \pm 1.3 \mathrm{~km} \mathrm{~s}^{-1}$, which corresponds to an estimated mass of the clump of $2000 M_{\odot}$. We checked that the signal is not resolved spatially as the integrated line in a single pixel or in the region of interest is about the same within the error bars. We thus assume that they have a typical projected size of $R_{\text {app }}<5.2 \mathrm{pc}(F W H M / 2)$ corresponding to the beam size or a root-mean-squared (RMS) spatial size of 
Table 1. Detection of one small clump.

\begin{tabular}{cccccccccc}
\hline \hline $\begin{array}{c}\text { RA } \\
(\mathrm{J} 2000)\end{array}$ & $\begin{array}{c}\text { Dec } \\
(\mathrm{J} 2000)\end{array}$ & $\begin{array}{c}\Delta \alpha \\
(\prime)\end{array}$ & $\begin{array}{c}\Delta \delta \\
\left({ }^{\prime \prime}\right)\end{array}$ & $\begin{array}{c}V_{\mathrm{LSR}} \\
\left(\mathrm{km} \mathrm{s}^{-1}\right)\end{array}$ & $\begin{array}{c}\Delta V \\
\left(\mathrm{~km} \mathrm{~s}^{-1}\right)\end{array}$ & $\begin{array}{c}S_{\max } \\
\left(\mathrm{mJy}^{2}\right)\end{array}$ & $\begin{array}{c}S_{\mathrm{CO} \Delta v} \\
\left(\mathrm{Jy} \mathrm{km} \mathrm{s}^{-1}\right)\end{array}$ & $\begin{array}{c}L_{\mathrm{CO}}^{\prime} \\
\left(\mathrm{K} \mathrm{km} \mathrm{s}^{-1} \mathrm{pc}^{2}\right)\end{array}$ & $\begin{array}{c}M_{\mathrm{H}_{2}} \\
\left(M_{\odot}\right)\end{array}$ \\
\hline $004244.52+411610.1$ & 1.7 & 1.7 & $-176 . \pm 1$. & $14 . \pm 3$. & 22.2 & $0.32 \pm 0.05$ & $480 \pm 70$ & $2000 \pm 300$ \\
\hline
\end{tabular}

Notes. We provide the $\mathrm{J} 2000$ coordinates, offsets, the number of detected pixels, the central velocity $V_{\mathrm{LSR}}$, the FWHM $\Delta V$, the peak intensity $S_{\text {max }}$, the integrated line $S_{\mathrm{CO}} \Delta v$, the line luminosity $L_{\mathrm{CO}}^{\prime}$, and the molecular mass derived for the clump detected close to the centre (in projection). The offsets here and throughout this Letter are computed with respect to the optical nucleus (J2000: $00 \mathrm{~h} 42^{\mathrm{m}} 44.37^{\circ}+41^{\circ} 16^{\prime} 8.34^{\prime \prime}$ ) defined by de Vaucouleurs \& Corwin (1985, see Fig. 6).

$\sigma_{r}<4.6$ pc. Fig. 4 displays the channel map corresponding to this signal, while Fig. 5 displays its spectra. In the following, we consider these upper values to discuss qualitative properties of this clump.

According to the Galactic velocity dispersion-size relation of Solomon et al. (1987), this clump lies above the $\sigma /\left(1 \mathrm{~km} \mathrm{~s}^{-1}\right)=$ $(R /(1 \mathrm{pc}))^{0.5}$ relation. It has an apparent mass surface brightness $21 M_{\odot} / \mathrm{pc}^{2}$ (computed within the FWHM area). It also lies well above the correlation between $\sigma_{v} / R^{1 / 2}$ and $\Sigma$ discussed by Heyer et al. (2009). With these characteristics, one can question if a $2000 M_{\odot}$ clump is gravitationally bound at a distance $D=9.1 \mathrm{pc}$ from the black hole. It should have a density $n_{\text {tide }}=\frac{M_{\mathrm{BH}}}{4 / 3 \pi D^{3} m_{\mathrm{p}}}>4.2 \times 10^{6} \mathrm{~cm}^{-3}$ to resist the tidal force from the supermassive black hole (Chen et al. 2016). This is much higher than the apparent mean density $n_{\text {app }} \sim\left(M_{\mathrm{H}_{2}} / m_{\mathrm{P}}\right) /\left[4 / 3 \pi R_{\text {app }}^{3}\right] \sim$ $150 \mathrm{~cm}^{-3}$. This would correspond to a volume filling factor smaller than $3.5 \times 10^{-5}$, which is well below the volume filling factor $(\sim 1 \%)$ estimated in Melchior \& Combes (2016) in the gas present along the minor axis in the inner bulge. This suggests that the gas is clumped at a smaller scale, as observed in the Galaxy (e.g. Combes 2001; Heyer \& Dame 2015). This would correspond to a clump size smaller than $R_{\text {app }}^{\prime}=0.24 \mathrm{pc}$. In addition, one can note that the $\mathrm{CO}$ luminosity and derived mass are significantly lower than the main clumps observed in the Central Molecular Zone (e.g. Oka et al. 2001).

Last, one can consider constraints from the kinematics. A cloud in the gravitational field of the black hole would have a circular velocity of $360 \mathrm{~km} \mathrm{~s}^{-1}$ : if one considers an inclination of $55 \mathrm{deg}$ (Bacon et al. 2001), one would expect a velocity of $200 \mathrm{~km} \mathrm{~s}^{-1}$. If there are non-circular motions, this is compatible with the amplitude of our value of $-176 \mathrm{~km} \mathrm{~s}^{-1}$. However, we would expect a velocity gradient within the beam of about $120 \mathrm{~km} \mathrm{~s}^{-1}$, while we detect a velocity dispersion of $13 \mathrm{~km} \mathrm{~s}^{-1}$. Figure 6 shows the location of the clump discussed here superimposed on an HST colour image (336-275 nm) with respect to the position of the so-called $\mathrm{P} 1, \mathrm{P} 2$, and $\mathrm{P} 3$ components.

These are convincing signs that this clump is probably not exactly in the mid-plane: if it were at $100 \mathrm{pc}$ along the line of sight, this gas clump would have the $\mathrm{CO}$ critical density and a filling factor of $1 \%$. Then, it would not be in the sphere of influence of the black hole, and its real size and velocity dispersion are probably below our spectral and spatial resolutions.

Rosolowsky \& Leroy (2006) discussed that diffuse emission can be missed as a result of interferometric filtering, but here we expect clumpy gas in this region with little filtering. In addition, these authors discussed that stable recovery of cloud properties can be achieved with a signal-to-noise ratio of at least 10 , while we reach 6.6. It is thus difficult to push this type of analysis further.

\subsection{Comparison with properties derived from infrared data}

Figure 3 shows that there is a deficit of $100 \mu \mathrm{m}$ emission next to the centre, in the region surrounding the black hole. Given the lack of resolution of infrared data, dust emission, which seems present in some maps in the near- and mid-infrared (Smith et al. 2012; Planck Collaboration Int. XXV 2015), corresponds to the emission in the central region, as displayed in Fig. 3. Viaene et al. (2014) estimated a dust mass of $475 M_{\odot}$ in a $36^{\prime \prime} \times 36^{\prime \prime}$ pixel, which is 157 times higher than the $\mathrm{PdB}$ beam. The stellar continuum is also very strong next to the centre: Viaene et al. (2014) computed a stellar mass of $3.71 \times 10^{8} \mathrm{M}_{\odot}$ in the same region. This central stellar component accounts for the central dust heating, as discussed by Groves et al. (2012). This stellar mass corresponds to old stars and should produce some supernova Ia (SNIa) every $100 \mathrm{yr}$ (Li et al. 2011b). Sjouwerman \& Dickel (2001) have detected four supernovae remnants. These supernovae might maintain supersonic turbulence (Mac Low \& Klessen 2004) and have contributed to expel the gas from the central region.

\section{Discussion}

We have detected one molecular gas clump with an estimated mass of $2000 M_{\odot}$ within $2.4^{\prime \prime}$ (9 pc) from the centre. Figure 6 displays a superposition of the central CO clump intensity on the 336-275 $\mathrm{nm}$ PHAT/HST image of the central region. While our molecular gas is at the position angle of the P1-P2 disc, it is blueshifted, while the stellar disc is redshifted. As discussed in Melchior \& Combes (2011), it could be rotating in a different orbit, while it could also be not exactly in the mid-plane and belong to a more distant component like the inner disc or inner ring. In addition, as argued in Sect. 3.2, the detected velocity dispersion is incompatible with the velocity gradient expected in the gravitation field of a large black hole. We conclude that this clump is outside the sphere of influence of the black hole.

The upper limit we place on the mass of the molecular gas next to the black hole is not compatible with the expectations from stellar mass loss that is due to the inner eccentric stellar disc. Chang et al. (2007) predicted a gas mass of $10^{4} M_{\odot}$ as a result of stellar mass loss and a $\mathrm{CO}(1-0)$ signal of $2 \mathrm{mJy}$ and a line width of $1000 \mathrm{~km} \mathrm{~s}^{-1}$ within $1 \mathrm{pc}$ of the black hole. Our observations exclude it at the level of $8.8 \sigma$. Given the simultaneous absence of dust emission (Viaene et al. 2014) and of molecular gas (this work), we can argue that the stellar feedback produced by the inner eccentric stellar disc population does not accumulate next to the black hole. Their model expects a compact source, which should have been detected by our interferometric observations. We can then question whether their proposed mechanism to produce the P3 star cluster is valid. Our result confirms that the current absence of star formation next to the black hole (e.g. Rosenfield et al. 2012; Groves et al. 2012) 


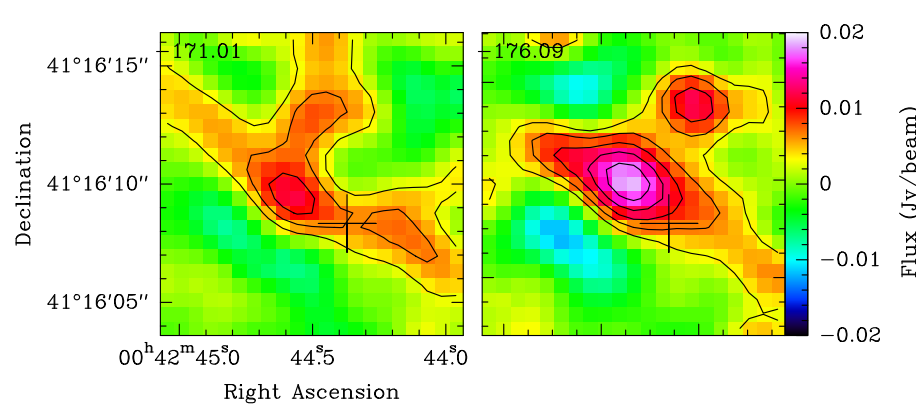

Fig. 4. Adjacent channel maps of the clump detected at $2.4^{\prime \prime}$ $(9.1 \mathrm{pc})$ from the centre. The contours are represented with a step of $3.2 \mathrm{mJy} /$ beam, namely $3.2,6.49 .6,12.8$, and $16 \mathrm{mJy} / \mathrm{beam}$. The cross indicates the position of the optical centre. If it is not an outlier, seen close to the centre only in projection, it lies inside the sphere of influence of the black hole.

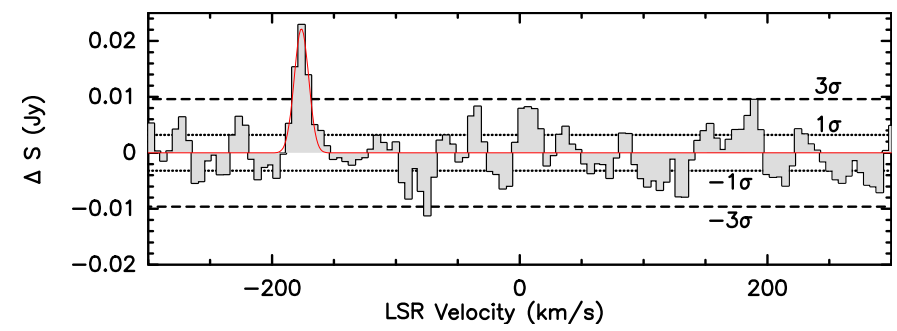

Fig. 5. Spectrum of the possible detection at $9.1 \mathrm{pc}$, potentially within the sphere of influence of the black hole. The systemic velocity of M31 $\left(-300 \mathrm{~km} \mathrm{~s}^{-1}\right)$ has been removed.

is due to the lack of gas. As discussed in Melchior \& Combes (2011), the scenario of a frontal collision with M32 as proposed by Block et al. (2006) could account for the two-ring morphology as well as for the absence of gas in the centre. In addition, the presence of a bipolar outflow of soft X-ray emission, detected along the minor axis by Li \& Wang (2007), Bogdán \& Gilfanov (2008) and possibly triggered by supernova explosions, could account for part of the missing gas.

Acknowledgements. Based on observations carried out with the IRAM Plateau de Bure Interferometer. IRAM is supported by INSU/CNRS (France), MPG (Germany), and IGN (Spain). We acknowledge the IRAM Plateau de Bure team for the observations. We thank Sabine Kœnig for her support with the data reduction. This project has benefited from support from the Programme National Cosmologie et Galaxies, Specific Action ALMA from INSU and Action Fédératrice Cosmologie et Structuration de l'Univers from the Paris Observatory. We are most grateful to the anonymous referee for the very constructive comments that helped us to substantially improve the manuscript. Special thanks go to Nelson Caldwell for providing a PHAT extract of the central field of Andromeda.

\section{References}

Amiri, N., \& Darling, J. 2016, ApJ, 826, 136

Azimlu, M., Marciniak, R., \& Barmby, P. 2011, AJ, 142, 139

Bacon, R., Emsellem, E., Combes, F., et al. 2001, A\&A, 371, 409

Belfiore, F., Maiolino, R., Maraston, C., et al. 2016, MNRAS, 461, 3111

Bender, R., Kormendy, J., Bower, G., et al. 2005, ApJ, 631, 280

Block, D. L., Bournaud, F., Combes, F., et al. 2006, Nature, 443, 832

Bogdán, Á., \& Gilfanov, M. 2008, MNRAS, 388, 56

Chang, P., Murray-Clay, R., Chiang, E., \& Quataert, E. 2007, ApJ, 668, 236

Chen, X., Amaro-Seoane, P., \& Cuadra, J. 2016, ApJ, 819, 138

Combes, F. 2001, Astro. Space Sci. Suppl., 277, 29

Crane, P. C., Dickel, J. R., \& Cowan, J. J. 1992, ApJ, 390, L9

Dahmen, G., Huttemeister, S., Wilson, T. L., \& Mauersberger, R. 1998, A\&A, 331,959

Dalcanton, J. J., Williams, B. F., Lang, D., et al. 2012, ApJS, 200, 18

de Vaucouleurs, G., \& Corwin, H. G., Jr. 1985, ApJ, 295, 287

Ford, G. P., Gear, W. K., Smith, M. W. L., et al. 2013, ApJ, 769, 55

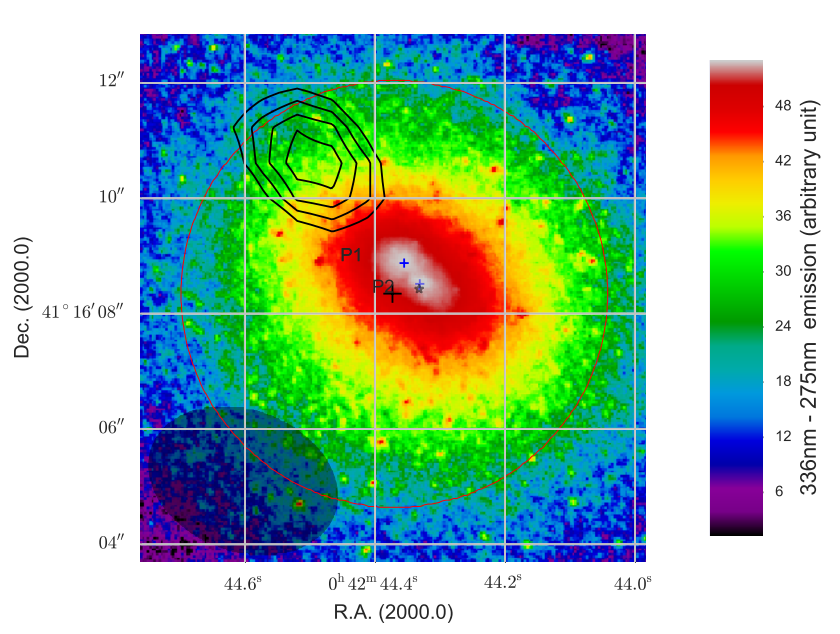

Fig. 6. $336 \mathrm{~nm}$ - 275 nm PHAT map (Dalcanton et al. 2012): the blue crosses correspond to the position of the so-called P1 and P2, the two maxima of this region (Bender et al. 2005). The black cross corresponds to our reference point, quoted by Crane et al. (1992) as the "optical nucleus" from de Vaucouleurs \& Corwin (1985). The star refers to the radio nucleus detected by Crane et al. (1992): it is in good agreement with the P2 position. In the bottom left corner, we show the IRAM$\mathrm{PdB}$ beam of our observations. The black contours correspond to our detection within the sphere of influence of the black hole (red circle): we summed the three main channels and display the contours above

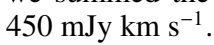

Gallagher, J. S., \& Hunter, D. A. 1981, AJ, 86, 1312

González-Martín, O., Masegosa, J., Márquez, I., et al. 2015, A\&A, 578, A74 Groves, B., Krause, O., Sandstrom, K., et al. 2012, MNRAS, 426, 892 Heckman, T. M. 1996, The Physics of Liners in View of Recent Observations, ASP Conf. Ser., 103, 241

Heyer, M., \& Dame, T. M. 2015, ARA\&A, 53, 583

Heyer, M., Krawczyk, C., Duval, J., \& Jackson, J. M. 2009, ApJ, 699, 1092

Jacoby, G. H., Ford, H., \& Ciardullo, R. 1985, ApJ, 290, 136

Jin, S.-W., Gu, Q., Huang, S., Shi, Y., \& Feng, L.-L. 2014, ApJ, 787, 63

Jedrzejewski, R. I. 1987, MNRAS, 226, 747

Kang, Y., Rey, S.-C., Bianchi, L., et al. 2012, ApJS, 199, 37

Kauffmann, J., Pillai, T., Zhang, Q., et al. 2017, A\&A, 603, A89

Lauer, T. R., Bender, R., Kormendy, J., Rosenfield, P., \& Green, R. F. 2012, ApJ, 745,121

Li, Z., \& Wang, Q. D. 2007, ApJ, 668, L39

Li, Z., Garcia, M. R., Forman, W. R., et al. 2011a, ApJ, 728, L10

Li, W., Chornock, R., Leaman, J., et al. 2011b, MNRAS, 412, 1473

Mac Low, M.-M., \& Klessen, R. S. 2004, Rev. Mod. Phys., 76, 125

Melchior, A.-L., \& Combes, F. 2011, A\&A, 536, A52

Melchior, A.-L., \& Combes, F. 2013, A\&A, 549, A27

Melchior, A.-L., \& Combes, F. 2016, A\&A, 585, A44

Melchior, A.-L., Viallefond, F., Guélin, M., \& Neininger, N. 2000, MNRAS, 312 , L29

Menezes, R. B., Steiner, J. E., \& Ricci, T. V. 2013, ApJ, 762, L29

Mutch, S. J., Croton, D. J., \& Poole, G. B. 2011, ApJ, 736, 84

Oka, T., Hasegawa, T., Sato, F., et al. 2001, ApJ, 562, 348

Planck Collaboration Int. XXV 2015, A\&A, 582, A28

Rahmani, S., Lianou, S., \& Barmby, P. 2016, MNRAS, 456, 4128

Ricci, T. V., Steiner, J. E., \& Menezes, R. B. 2015, MNRAS, 451, 3728

Rimoldi, A., Rossi, E. M., Costantini, E., \& Portegies Zwart, S. 2016, MNRAS, 456, 2537

Rosenfield, P., Johnson, L. C., Girardi, L., et al. 2012, ApJ, 755, 131

Rosolowsky, E., \& Leroy, A. 2006, PASP, 118, 590

Rosolowsky, E., \& Leroy, A. 2011, Astrophysics Source Code Library, [record ascl: 1102.012$]$

Rubin, V. C., \& Ford, W. K., Jr. 1971, ApJ, 170, 25

Sjouwerman, L. O., \& Dickel, J. R. 2001, Young Supernova Remnants, AIP Conf. Proc. 565, 433

Smith, M. W. L., Eales, S. A., Gomez, H. L., et al. 2012, ApJ, 756, 40

Solomon, P. M., \& Vanden Bout, P. A. 2005, ARA\&A, 43, 677

Solomon, P. M., Rivolo, A. R., Barrett, J., \& Yahil, A. 1987, ApJ, 319, 730

Tempel, E., Tuvikene, T., Tamm, A., \& Tenjes, P. 2011, A\&A, 526, A155

Viaene, S., Fritz, J., Baes, M., et al. 2014, A\&A, 567, A71 